\title{
LA ESTIMACION INDIRECTA \\ DE LA PRODUCCION AGRARIA \\ EN EL SIGLO XIX: \\ REPLICA A SIMPSON
}

LEANDRO PRADOS DE LA ESCOSURA

Universidad de Cantabria

James Simpson ha escrito un interesante artículo sobre las tendencias del consumo de alimentos y de la producción agraria (en el núm. VII, 2, de esta Revista). Uno de los aspectos más destacables del mismo es la audacia con que Simpson aborda el espinoso problema de la medición indirecta del producto de la agricultura ante la ausencia de fuentes homologables a los diezmos o a las modernas estadísticas. Comparto con él el interés por conocer las grandes líneas de la evolución del sector agrario a lo largo del siglo XIX, pues, de otro modo, resultará difícil establecer conjetura razonable alguna sobre el cambio económico en el conjunto de la economía española. Mi réplica a su artículo la suscita, sin embargo, el desacuerdo con el método indirecto propuesto para reconstruir las tendencias de la producción agraria, así como con los resultados derivados de éste.

Tres son los aspectos que Simpson considera en su artículo. En primer lugar, intenta demostrar la escasa fiabilidad de las fuentes existentes para establecer los cambios a largo plazo en la producción agraria y concluye abogando por los métodos de estimación indirecta del producto de la agricultura. En segundo lugar, analiza la composición de la dieta mediante la construcción de una «hoja de balance» para 1900 y 1930, lo que le permite deducir que la dieta era baja en calorías (2.100-2.500 por persona/día) y que estaba mayoritariamente compuesta de productos básicos. Asimismo, infiere de la pobre dieta de 1900 que ésta debió haber sido la misma a lo largo del siglo XIX, pues no le parece plausible que la población en años normales consumiese una cantidad de calorías inferior por persona y día. Por último, lleva a cabo una estimación del consumo y del producto agrario por decenios para el siglo $\mathrm{xIX}$.

La estimación del consumo está basada en el supuesto de un consumo calórico constante por persona entre 1800 y 1900 (derivado del razonamiento ofrecido en la sección anterior del artículo) que identifica con un consumo estable de productos agrarios por habitante. El consumo por persona multiplicado por el número de habitantes permite alcanzar la cifra global del 
consumo de mercancías agrarias. Deduciendo de esta cifra las importaciones y añadiendo las exportaciones, Simpson obtiene el producto agrario. Sus resultados muestran un crecimiento de la producción ligeramente superior al de la población pero no al de la mano de obra agraria, con lo cual la productividad (medida como el producto medio por trabajador) no habría variado en la agricultura española a lo largo del siglo xix. La proporción invariable de mano de obra empleada en la agricultura y el pequeño avance relativo de los cultivos de alta rentabilidad (vinculados al dinámico pero reducido sector exterior) no hicieron posible la elevación del producto medio por trabajador en un contexto caracterizado por la ausencia de cambio técnico y el déficit de abonos.

En síntesis, la interpretación de Simpson rechaza la visión más optimista propuesta por Prados y reafirma la expuesta por Tortella: el crecimiento intensivo de la agricultura es, pues, un fenómeno del siglo $\mathbf{x x}$.

A su último apartado dedicaré mi comentario. Para ello, distinguiré varios aspectos.

\section{La critica de las fuente}

Simpson dedica el primer apartado de su artículo a poner de relieve la falta de fiabilidad de las fuentes sembrando dudas sobre su calidad. Así, el Censo de Frutos y Manufacturas es rechazado citando testimonios contemporáneos que apuntan a una subestimación de las cifras de producción de cereales, así como mediante la observación de la «relativa estabilidad» de la producción de trigo para los cinco años de que se tiene noticia entre 1787 y 1803. Simpson recela de la estabilidad de las cifras de producción de cereales en estos años (el coeficiente de variación es de 4,6 por 100, frente al 12,6 por 100 en 1919-35) y sugiere que está en contradicción con las fuertes fluctuaciones de precios en estos años. Sin embargo, esta observación aparentemente atinada no es tan convincente si se repara en la construcción del cuadro 1 que Simpson explica en una nota aneja. Resulta obvio que adoptar la media de otros años en ausencia de información para un año concreto invalida el razonamiento de Simpson. Llama la atención, por otra parte, que Simpson no lleve a cabo test alguno para comprobar sus sospechas. Así, si suponemos que no hubo cambios apreciables en la renta real por habitante, y aceptamos que el crecimiento de la población en el periodo 1787-1803 fue idéntico al de $1787-1797(0,14$ por 100 anual $)$, y que el saldo equilibrado de la balanza comercial agraria en 1792 es representativo de todo el período, la tasa de variación anual de la demanda de pro-

ductos agrarios sería igual a la de la producción y, por tanto, equivaldría 
a la tasa de crecimiento demográfico más la tasa de variación de los precios relativos de los cereales ponderada por la elasticidad-precio de su demanda. Dado que esta última puede situarse entre $-0,3$ y $-0,5$, y que existe abundante información acerca del movimiento de los precios en estos años, Simpson podría haber contrastado su hipótesis.

La falta de concordancia entre distintas fuentes acerca de las cifras de superficie cultivada de cereales en la década de 1890 , y el deficiente método de cálculo de la producción por unidad de superficie empleado por los coetáneos, conducen a Simpson a rechazar la fiabilidad de las estadísticas de finales del siglo xix. Sorprende que no lleve a cabo el autor comprobación alguna del grado de plausibilidad de dichas estimaciones. Como recientemente nos ha recordado Crafts, a diferencia de los economistas, los histo- riadores económicos nos vemos obligados a trabajar con datos de calidad dudosa y a dedicar un considerable esfuerzo a la construcción de nuevas bases de datos '. Por ello, me llama la atención la ligereza con que Simpson rechaza la información cuantitativa disponible.

\section{El método para calcular el producto agrario}

Simpson rechaza mis estimaciones del consumo y la producción de la agricultura obtenidas mediante el enfoque de demanda arguyendo que en mi argumento existe circularidad, pues utilizo la renta nacional para calcular. la producción agraria; tiendo, además, a identificar los cambios en la demanda con los de la producción, y no tomo en cuenta las variaciones en la distribución de la renta. Dados los defectos del modelo y la escasa fiabilidad de las fuentes empleadas para especificar los parámetros que aparecen en la parte derecha de la ecuación de demanda (así como mi decisión de preferir los niveles estimados más altos por coincidir con los resultados derivados de las fuentes contemporáneas), Simpson concluye que mis resultados son rechazables. No voy a defender mis estimaciones, pues han sido publicadas para provocar el debate. Acerca de las fuentes sólo deseo puntualizar que, en mi opinión, la precisión exigible a las fuentes depende de aquello que se trata de demostrar; es decir, la cuestión a dirimir sería qué grado de error en las fuentes invalida los resultados de mi ejercicio analítico. Sí he de precisar, no obstante, que Simpson malinterpreta el propósito de mi estudio: se trataba de un test de consistencia de las cifras de producción agraria derivados de las fuentes coetáneas, $y$, por tanto, la crítica de circularidad no es pertinente; $y$, por supuesto, nunca dije que producción y demanda fueran una

\footnotetext{
' Crafts (1987), p. 174.
} 
misma cosa. Finalmente, resulta curioso que para Simpson (p. 375) los resultados de mi estimación indirecta para 1800-1890 no parezcan inaceptables, y rechace seguidamente aquéllos para los años 1890-1910, que coinciden con los obtenidos por el GEHR con métodos directos ${ }^{2}$.

Pese a la crítica, Simpson elige la vía indirecta para estimar el consumo ante la falta de información fidedigna sobre la superficie cultivada y los rendimientos físicos. A fin de establecer el consumo de alimentos por habitante, elabora una hoja de balance para 1900 que, con objeto de homogeneizar en una unidad común, convierte en calorías (y no, sorprendentemente, en valor mediante sus precios de mercado). La cifra final oscila entre 2.100 y 2.500 calorías/habitante/día, cifra que considera plausible tras contrastarla con las de otros países. La dieta es poco variada, como revela el hecho de que cereales, leguminosas y patatas constituyan el 64 por 100 del consumo total de calorías (el 60 por 100 en 1931 (p. 369). Simpson apunta, además, que existieron diferencias regionales y cambios a largo plazo en la dieta (expansión del consumo de la patata y del aceite de oliva y aumento del consumo de productos no elaborados en la región gracias a la mejora en el transporte), así como diferencias en la calidad de la dieta entre clases sociales. Sin embargo, señala Simpson (p. 378), «aunque hubo cambios en la dieta durante el siglo, nuestra cifra de consumo de aproximadamente 2.100 calorías ( 2.500 brutas), probablemente no fue muy diferente a la cifra en 1800 ». La principal razón que subyace tras este supuesto es que el nivel de consumo calórico era tan reducido hacia 1900 que difícilmente podría haber subsistido la población por debajo de él en épocas anteriores. Simpson advierte, no obstante, que un nivel de consumo por habitante constante en calorías en dos fechas distintas no significa estancamiento (p. 378). Pese a esta importante y necesaria matización, en el párrafo siguiente Simpson afirma (p. 379): "Si se supone una cifra media de 2.100 calorías/habitante/día para el siglo XIx, la producción a largo plazo cambiaría al mismo ritmo y dirección de la población, corregido por las cifras de importación y exportación de alimentos.» De este modo, Simpson calcula el valor del consumo por persona a partir de las cifras del GEHR (1983) en 1900, las multiplica por la población de cada decenio a lo largo del siglo xix, y a continuación aplica a esta estimación del valor del consumo a precio de 1900 un índice de precios del trigo para convertirlos en valores a precios corrientes, les añade las exportaciones netas, y, finalmente, tras deflactar el resultado con el índice de precios del trigo, obtiene sus estimaciones del producto agrario entre 1800-1809 y 1890-1899.

El paso que realiza Simpson de identificar un número de calorías cons-

\footnotetext{
2 GEHR (1987).
} 
tante por persona/día con un consumo estable de productos agrarios por habitante representa, desde el punto de vista económico, la aceptación implícita de que, entre 1800 y 1900 , el invariable volumen de calorías consumidas por individuo procede del mismo conjunto de productos de la agricultura, y en proporciones idénticas. Naturalmente, aceptar que el consumo calórico fuese aproximadamente el mismo a lo largo del siglo xIX resulta mucho más fácil que admitir que no tuvieron lugar alteraciones en la composición del consumo per capita y que, dada la escasa entidad relativa de las exportaciones netas, el producto agrario mantuviese, en consecuencia, una composición prácticamente igual en 1800 y 1900 . La razón de esta diferencia se encuentra contenida en la propia argumentación de Simpson: se pueden consumir las mismas calorías por individuo y, sin embargo, variar la composición del consumo con una mayor participación de productos con un valor unitario superior; esto es, de mayor calidad. Simpson podría argüir que tales cambios no se produjeron y, por ello, un sencillo ejercicio aritmético, que, además, se puede situar históricamente, servirá para ilustrar lo que intento decir.

He valorado las cantidades consumidas en 1900 de un conjunto de productos que representaba el 73,5 por 100 de las calorías $(670.000)$ consumidas por individuo (pan de trigo, carne de vaca, oveja y caprino, pescado -bacalao-, patatas, garbanzos, arroz, vino, azúcar y aceite) con sus precios al por menor en $1913^{3}$. El resultado es de 155 pesetas por individuo. Supongamos ahora que tuviese lugar una elevación de un 25 por 100 en el consumo de harina de trigo, esto es, que se elevase el consumo a $131,9 \mathrm{Kg}$./habitante al año (lo que representaría $175,9 \mathrm{Kg}$. de trigo, neto de simiente, con los coeficientes empleados por Simpson). Esta cifra resultaría plausible para la primera mitad del siglo xIX a tenor de las cifras coetáneas apuntadas por Simpson para 1799 y 1868 . Manteniendo, como propone Simpson, el mismo consumo calórico, esta alteración en la composición del consumo permitiría sustituir por trigo otros productos hasta un volumen de 90.219 calorías (el equivalente de $26,4 \mathrm{Kg}$. de harina de trigo). Por otra parte, a precios de $1913,1.000$ calorías de trigo valían 0,117 pesetas, mientras 1.000 calorías de los restantes productos antes mencionados suponían, por término medio, 0,3651 pesetas. Así, pues, la sustitución de 90.219 calorías de este conjunto de productos por harina de trigo representaría un abaratamiento de 22,4 pesetas/habitante $[(0,3651-0,117) \times 90.219]$. El nuevo coste de las 670.000 calorías por individuo/año sería ahora 132,6 pesetas, es decir, un 85,5 por 100 de su valor anterior.

Consideremos ahora dos fechas históricas con idéntico consumo calórico

${ }^{3}$ Anuario Estadistico (1923). 
por persona/día: 1850-1859 y 1890-1899. De acuerdo con los cálculos de Simpson, el producto agrario se habría elevado un 29,4 por 100 entre ambas fechas. Si suponemos ahora que, manteniendo el mismo consumo de calorías, la proporción de trigo hubiese sido superior en un 25 por 100 en la primera fecha (como podría sugerirse a partir de las cifras citadas por Simpson para la primera mitad del siglo xIX), el producto agrario habría crecido de manera más intensa: en un 51 por 100 (a precios de 1913).

Se observa, pues, que la inferencia que realiza Simpson del consumo constante por persona de productos agrarios durante el siglo xIx a partir de su hipótesis, quizá plausible pero no demostrada, de un consumo igual de calorías/habitante/día no resulta aceptable y que su método, por consiguiente, no es válido para estimar el producto agrario de manera indirecta.

\section{Análisis de consistencia de los resultados}

El tercer y último aspecto de la comunicación de Simpson que tomaré en consideración son sus resultados para el crecimiento del producto agrario: 0,64 por 100 (con un límite superior de 0,76 por 100) anual, y la consecuencia que se deriva de ellos: el estancamiento de la productividad de la mano de obra (expresada como el producto medio por trabajador). Las interrogantes que plantean tales conclusiones son:

1) ¿Cómo es posible conciliar un crecimiento extensivo en la agricultura, esto es, sin cambios en el producto por trabajador, con la expansión industrial que tuvo lugar en España durante el siglo xix [Carreras (1984), Prados (1988)]?

2) ¿Cuál sería la condición exigida para que manteniéndose inalterado el porcentaje de mano de obra empleado en la agricultura se elevase el producto por trabajador en el sector?

III.1. Con objeto de observar la consistencia entre los resultados pro. puestos por Simpson para el crecimiento del consumo y de la producción de productos agrarios y la generalmente aceptada expansión de la producción industrial por persona y por trabajador a lo largo del siglo xIx, he llevado a cabo los pasos siguientes:

a) En primer lugar, he calculado el crecimiento de la renta per capita que implícitamente se deduce del supuesto de Simpson acerca de un crecimiento de la demanda igual al de la población a partir de una función de la demanda de alimentos:

$$
D_{a}=f\left(N, y, R P_{a}\right)
$$


donde $N$ representa a la población, $R P_{a}$ a los precios relativos e $y$ a la renta per capita. Si se toman en consideración las variaciones de la demanda,

$$
\pi D_{a}=\pi N+\eta \pi y+\varepsilon \pi R P_{a}
$$

siendo $\pi$ tasas de variación anual, $\eta$ la elasticidad de la demanda con respecto a la renta y $\varepsilon$ la elasticidad con respecto a los precios.

De [2] puede deducirse:

$$
\pi y=1 / \eta\left(\pi D_{a}-\pi N=\varepsilon \pi R P_{a}\right)
$$

y si suponemos con Simpson que el incremento de la demanda de productos agrarios equivale al de la población, $\pi D_{a}=\pi N$, tendremos:

$$
\pi y=-\varepsilon \pi R P_{a} / \eta
$$

Podemos calcular de manera aproximada los cambios en los precios relativos agrarios, mientras que para un país como España en el siglo XIX los valores de las elasticidades renta y precios de la demanda de alimentos fluctuarían de 0,7 a 1,0 y de $-0,2$ a $-0,6$, respectivamente ${ }^{4}$.

He procedido, pues, a calcular, a partir de [4], límites máximos y mínimos del crecimiento del ingreso real por habitante que fuesen compatibles con un crecimiento anual de la demanda de alimentos idéntico al de la población entre 1800 y 1890 , así como entre 1800 y 1910. Los resultados que arroja este ejercicio muestran que la renta real per capita habría crecido entre 0,09 y 0,38 por 100 anualmente durante el período $1800-1890$, y entre 0,07 y 0,31 por 100 durante $1800-1910$.

¿Son compatibles tales tasas de crecimiento del ingreso real por habitante con la expansión industrial que aceptan hoy día los historiadores? Un test para contrastar esta hipótesis puede llevarse a cabo mediante el recurso de nuevo a una función de demanda de productos industriales que, expresada en términos de variación anual, sería igual a:

$$
\pi D_{i}=\pi N+\mu_{\pi y}+\sigma \pi R P_{i}
$$

siendo $\mu$ y $\sigma$ las elasticidades renta y precios de la demanda de productos industriales $(i)$.

4 Cfr. Prados de la Escosura (1988), cap. 3. Chenery y Syrquin (1980) llegan a postular 0,4 para países en desarrollo actuales con un nivel de ingreso por habitante similar, pero para Europa en el siglo xIx resulta un valor muy bajo. 
Mi reciente estimación del crecimiento de la producción industrial, que aminora la expansión sugerida por Carreras, permite obtener, junto con el saldo de la balanza comercial de productos manufacturados, una estimación del crecimiento de la demanda de productos industriales: 1,59 por $100 \mathrm{y}$ 1,58 por 100 para $1800-1890$ y $1800-1910$, respectivamente ${ }^{5}$. No disponemos aún de un índice de precios industriales, pero podríamos suponer que los precios relativos industriales evolucionaron en sentido opuesto al de los precios relativos agrarios. Es decir, mientras que éstos aumentaron, aquéllos tendieron a declinar. La evolución de los precios de los productos agrarios y manufacturados en el comercio exterior sugieren que así fue ${ }^{6}$. Más difícil es precisar la intensidad de su declive y, por ello, he supuesto arbitrariamente que era igual a la del crecimiento de los precios relativos agrarios, es decir, un límite máximo (1800-1890: 0,45 por $100 ; 1800-1910: 0,36$ por 100).

No existen estimaciones de las elasticidades renta y precios de la demanda de productos industriales para la España del siglo xix. Chenery y Syrquin proporcionan valores para las elasticidades-renta de la demanda de una amplia gama de productos industriales para países a niveles de ingreso por habitante análogos (300-600 dólares USA de $1970 \mathrm{PPA})^{7}$. Si se ponderan las elasticidades-renta que corresponden a la demanda de los productos de las diferentes industrias por la participación de éstas en el «valor añadido» de la industria española hacia 1856 y 1900, resultaría posible obtener valores globales para la elasticidad-renta de la demanda de bienes manufacturados $^{8}$. Así, $\mu$ sería de 1,06 para 1856 y de 1,14 para 1900 . Chenery y Syrquin no proporcionan valores de la elasticidad-precio. Por su parte, Lluch, Powell y Williams ofrecen valores de las elasticidades renta y precio de la demanda de vestido (clothing) para dos tramos de renta per capita (dólares USA de 1970), 100-500 y 500-1.000, con valores de $\mu: 0,97$, y $\sigma:-0,3$, para el primero de ellos, y $\mu: 1,28 ; \sigma:-0,6$, para el segundo ${ }^{9}$. El valor estimado por Chenery y Syrquin para este sector industrial es de 1,014 (1970 dólares USA $300<y>600$ ). Un valor de 1,0 para $\mu$, resulta verosímil siendo 1,1 un valor máximo, mientras que para $\sigma$ un valor de $-0,3$ parece aceptable. Sin embargo, he sesgado al alza los valores de ambas elasticidades con objeto de obtener el menor valor plausible para el crecimiento de la renta real per capita (esto es, compatible con el de la demanda de productos industriales), y para ello, he tomado valores de $1,1-1,2$ para $\mu$ y $-0,6$ para $\sigma$.

\footnotetext{
'Prados de la Escosura (1988), caps. 4 y 5; Carreras (1984).

- Prados de la Escosura (1982, 1985).

Chenery y Syrquin (1980).

- Prados de la Escosura (1988), cap. 4; Nadal (1987).

" Lluch, Powell y Williams (1977).
} 
Los resultados alcanzados para el crecimiento del ingreso real por habitante con la ecuación [5] son, para 1800-1890, 0,66-0,72, y para 1800-1910, 0,67-0,73 (según se adopten los valores de 1,2 ó 1,1 para la elasticidad-renta). Se trata, en ambos casos, de un crecimiento muy superior al que se deriva del supuesto de Simpson acerca de un consumo constante de productos agrarios por habitante.

Podría plantearse, pues, como caso extremo, una caída de los precios de los precios relativos no es admisible por falta de apoyo empírico, y, a continuación, postular que la caída de los precios relativos industriales habría sido muy superior, de modo que fuese compatible con un crecimiento mucho más lento del ingreso real por habitante: por ejemplo, el obtenido anteriormente bajo el supuesto de Simpson. Así, con el límite máximo de crecimiento que permite el supuesto de Simpson y con $\mu: 1,2$, la caída de los precios relativos industriales habría sido del $-1,0067$ por 100 anual entre 1800 y 1890 , es decir, para un nivel 100 en 1800 , en 1890 sería de 40,6 , mientras que el mismo ejercicio para el período 1800-1910 arrojaría un descenso de los precios relativos industriales del $-1,08$ por 100 anual, esto es, habría pasado a un nivel 30,7 en 1910 ( siendo $1800=100$ ). Semejante declive de los precios relativos industriales habrían exigido profundos cambios en la productividad de la industria española que, además, tendrían que haber sido traducidos en una reducción de los precios, y no en una mayor retribución de los factores de producción empleados en la industria. El modesto crecimiento de la productividad en la industria $(0,69$ por 100 anual entre 1800 y 1910 para el producto por trabajador) no parece haberlo permitido aún si suponemos que la productividad de la agricultura no varió (pero tampoco empeoró), como hace Simpson. Naturalmente, si se aceptan las hipótesis de algunos historiadores acerca del carácter oligopolístico de la industria manufacturera, la posibilidad de convertir la totalidad de los incrementos de productividad industrial en menores precios de los productos manufacturados estaría completamente fuera de lugar ${ }^{10}$.

Podría plantearse, pues, como caso extremo, una caída de los precios relativos industriales equivalente al aumento del producto industrial por trabajador empleado (tomado como índice de la productividad de la mano de obra), suponiendo que la productividad agraria se mantuviera estable a lo largo del período y que los cambios en la productividad industrial se traducían en cambios en los precios relativos de la misma intensidad aunque en sentido opuesto. Cualquier otra posible influencia sobre los precios relativos queda, por tanto, descartada. Así, pues, los precios relativos industriales habrian descendido a una tasa anual de $-0,44$ por 100 entre 1800 y 1890 , y

${ }^{10}$ Fraile (1985). 
de $-0,69$ por 100 entre 1800 y 1910 . Aceptando los valores máximos para $\mu$ y $\sigma$ de 1,2 y $-0,6$, respectivamente, la renta real por habitante habría crecido al 0,66 por $100 \mathrm{y}$ al 0,51 por 100 anualmente en los períodos 1800 . 1890 y $1800-1910$.

Nuevamente se desprende de este ejercicio que el supuesto de Simpson acerca de un consumo constante por habitante de productos agrarios no es consistente con la evidencia disponible para el conjunto de la economía española en el largo siglo xIx.

No puede concluirse este apartado sin estimar cuál pudo ser el crecimiento del consumo y la producción de productos agrarios durante el siglo XIX. En primer lugar, calcularé el crecimiento de la demanda de alimentos. Para ello, utilizaré la ecuación [1]. Las tasas de variación de la renta per capita son las derivadas de la demanda de productos industriales, que he redondeado en 0,7 para cada período. Los valores de las elasticidades fluctúan entre 0,7 y 1,0 para $\eta, y-0,2$ y $-0,6$ para $\varepsilon$. Los resultados muestran que, como mínimo, entre 1800 y 1890 , la demanda de alimentos creció a un 0,75 por 100 anual (esto es, cuando $\eta: 0,7$ y $\varepsilon:-0,6$ ), mientras que, entre 1800 y 1910 , lo haría al 0,83 por 100 . Este crecimiento de la demanda se traduciría (con los datos de Simpson para la balanza comercial agraria) en un crecimiento mínimo del producto agrario de 0,83 por 100 anualmente entre $1800 / 1809$ y $1890 / 1899$, y en un 0,87 por 100 entre $1800 / 1809$ y $1900 /$ 1909. Naturalmente, estas tasas de crecimiento de la producción agraria superan a las de la población $(0,53$ y 0,56 , respectivamente), y también ligeramente a las de la mano de obra masculina empleada en la agricultura $(0,78$ por 100 y 0,75 por 100 anual en $1800-1890$ y $1800-1910$ ).

Para la España del siglo xix, sin embargo, una elasticidad-precio de $-0,6$ para la demanda de productos agrarios resulta excesiva, puesto que revelaría una opción de alimentos variada. Las ideas expuestas por Simpson acerca de una dieta monótona y compuesta mayoritariamente de productos básicos sugeriría un valor absoluto menor. Así, he repetido el ejercicio manteniendo $\eta: 0,7$, y suponiendo valores más plausibles de la elasticidad-precio $(\varepsilon:-0,5$; $-0,4 ; y-0,3)$. Los resultados que éste arroja muestran que, para el período 1800/1809-1890/1899, la demanda habría crecido anualmente al 0,8,0,84 ó 0,88 por 100 , y la producción al $0,87,0,91$ ó 0,96 por 100 , tasas todas ellas superiores a la del crecimiento del número de activos masculinos en la agricultura $(0,78$ por 100$)$. Para el período $1800 / 1809-1900 / 1909$, los resultados serían, a su vez, de $0,87,0,91$ y 0,94 por 100 para la demanda de alimentos, y de $0,91,0,94$ y 0,98 por 100 para la producción agraria también superiores al crecimiento de la mano de obra agraria $(0,75$ por 100$)$.

En mi estudio, el propósito de la estimación de demanda era comprobar la consistencia entre el crecimiento del producto agrario que se podía derivar 
de datos contemporáneos dispersos y habitualmente considerados poco fiables, y lo que los historiadores habian ido aportando a la cuantificación de la modernización económica española en el siglo xix. Los datos coetáneos sugerían que el producto agrario debió crecer al 0,96 por 100 anual acumulativo entre 1800 y 1890 , y al 0,98 por 100 entre 1800 y 1910 . Mis resultados indirectos mostraban una cierta concordancia con la visión que se desprendía de las cifras coetáneas. Ahora, en esta nueva aproximación, la visión coetánea no sería ya el límite máximo que sugerían mis cálculos indirectos, sino que coincidiría plenamente con ellos.

Obviamente, este ejercicio no justifica el abandono de la investigación directa, sino que, por el contrario, tan sólo propone algunas hipótesis acerca de la evolución de la agricultura que estudios más detallados y con fuentes apropiadas (quizá los inventarios "post-mortem" arrojen alguna luz al respecto, como ha sucedido ya en la historia económica británica o norteamericana) habrán de corroborar y ampliar o rechazar.

III.2. La segunda parte del ejercicio intenta contestar a la objeción que Simpson plantea a mi sugerencia de que, a largo plazo, el producto agrario por trabajador se elevó. Dado que no tuvo lugar una mecanización apreciable de la agricultura durante el largo siglo xIx, el aumento de la productividad habría de depender, de acuerdo con Simpson, de la sustitución de «cosechas de relativamente bajo valor por otros más altos» o de la reducción relativa de la población activa agraria (p. 381). Con respecto a la primera posibilidad, Simpson identifica cambios hacia cultivos de más alta rentabilidad con la demanda externa y rechaza esta alternativa con el argumento de que el comercio exterior de productos agrarios adoleció de escaso peso relativo en la producción de la agricultura española. Esta identificación es básicamente correcta, pero dentro de la producción para el mercado interior también existieron posibilidades de transferir recursos hacia cultivos de mayor rentabilidad. Es el segundo aspecto, sin embargo, el que me parece más importante porque, aunque no es un argumento nuevo, aparece con un cierto tono científico. Mi propósito es mostrar cómo (hasta cierto punto) son compatibles el aumento del producto por trabajador en el sector agrario y el mantenimiento de la importancia relativa de la agricultura como empleadora de mano de obra. En otras palabras, voy a intentar apoyar la idea de que durante el largo siglo xix tuvo lugar un aumento del producto por trabajador en la agricultura mediante la utilización más intensa de trabajadores subempleados gran parte del año. Naturalmente, este aumento del producto medio por trabajador podría ser compatible con el mantenimiento del mismo nivel de producto por hora/hombre (esto es, con una productividad constante de la mano de obra). 
En su forma más sencilla, el enfoque de la función de producción permite expresar la tasa de variación del producto agrario como la suma de las tasas de variación de los distintos factores de producción ponderados por sus elasticidades con respecto al producto (que pueden aproximarse con sus correspondientes porcentajes en la renta nacional) más un residuo que se identifica con la productividad total de los factores (productividad global) ${ }^{11}$.

Así,

$$
\pi Q_{a}=\alpha \cdot \pi K_{a}+\beta \cdot \pi L_{a}+\tau \cdot \pi T+r
$$

donde $K, L$ y $T$ representan capital, trabajo y tierra, $\alpha, \beta$ y $\tau$, la proporción de la renta nacional que corresponde a beneficios, salarios y renta de la tierra, y $r$, la productividad global. El subfijo ${ }_{a}$ indica agricultura.

Si definimos

$$
\varphi=\alpha \cdot \pi K_{a}+\tau \cdot \pi T+r
$$

entonces, sustituyendo en [6],

$$
\pi Q_{a}=\varphi+\beta \cdot \pi L_{a}
$$

Por otra parte, podemos expresar:

$$
\pi Q_{a}=a \cdot \pi D_{a}
$$

y, de acuerdo con [2] y [8], sustituir ambos términos,

$$
\varphi+\beta \cdot \pi L_{a}=a\left(\pi N+\eta \cdot \pi y+\varepsilon \cdot \pi R P_{a}\right)
$$

siendo

$$
\pi N=b \cdot \pi L
$$

en donde $L$ es la población activa total.

De este modo,

$$
\pi L_{a}=1 / \beta\left(a b \cdot \pi L+a \eta \cdot \pi y+a \varepsilon \cdot \pi R P_{a}-\varphi\right)
$$

A fin de que la agricultura libere mano de obra, será preciso que el aumento de la mano de obra agraria sea inferior al del volumen total de fuerza de trabajo en la economía: $\pi L_{a}<\pi L$. Por tanto, ello exige que

$$
1 / \beta\left(a b \cdot \pi L+a \eta \cdot \pi y+a \varepsilon \cdot \pi R P_{a}-\varphi\right)<\pi L
$$

"En los párrafos que siguen me he inspirado en Crafts (1980). 
Esta expresión puede reordenarse del siguiente modo:

$$
\varphi-(a b-\beta) \pi L-a \eta \cdot \pi y-a \varepsilon \cdot \pi R P_{a}>0
$$

Naturalmente, para que disminuya la proporción de mano de obra empleada en el campo será preciso que

$$
\varphi>(a b-\beta) \pi L+a \eta \cdot \pi y+a \varepsilon \cdot R P_{a}
$$

Disponemos de información que nos permite estimar de manera aproximada el valor de la parte derecha de esta desigualdad para 1800-1910. La población activa creció en su conjunto a una tasa del 0,67 por 100 , mientras que en el apartado anterior he adoptado valores para las elasticidades $\left(\gamma_{1}: 0,7 ; \varepsilon:-0,5\right.$ a $\left.-0,3\right)$ y calculado el crecimiento de la renta per capita $(0,7$ por 100$)$. Los coeficientes $a$ y $b$ pueden obtenerse de las expresiones [9] y [11]. La única dificultad es $\beta$. Vandellós estimó, sin embargo, la participación de los salarios en la renta agraria para 1913: 0,523 ${ }^{12}$. Resulta, pues, lógico aceptar para $\beta$ un valor de, al menos, 0,6 para el siglo xIx, pues es plausible suponer que la participación de los salarios en la renta de la agricultura fuese inferior en 1913 que en 1800. Así, para que la participación de la mano de obra en las actividades agrarias disminuyese en términos relativos, se requeriría que $\varphi$ fuese mayor que 0,50/0,44 (dependiendo de que el valor adoptado para $\varepsilon$ sea $-0,3$ ó $-0,5)^{13}$.

A su vez, para que la población activa agraria disminuyera en términos absolutos se habría de cumplir en [12] que

$$
\varphi>a b \cdot \pi L+a \eta \cdot \pi y+a \varepsilon \cdot \pi R P_{a}
$$

Ello significaría que $\varphi$ alcanzara un valor de $0,904(\varepsilon:-0,3)(0,843$, si $\varepsilon: 0,5)^{14}$.

Veamos ahora qué valores alcanza $\varphi$ en la realidad. De acuerdo con la expresión [8], $\varphi=\pi Q_{a}-\beta \cdot \pi L_{a}$. Según los datos disponibles, la población activa masculina empleada en la agricultura creció en torno al 0,75 por 100 anual entre 1797 y $1910^{15}$. Así, aceptando un valor de 0,6 para la participación de los salarios en la renta agraria $(\beta)$, los valores de $\varphi$ serán de 0,53 ó

"Vandellós (1925).

${ }^{13}$ En el caso que los valores dados a $\eta$ y $\varepsilon$ fueran de 1,0 y $-0,3, \vartheta$ habría de ser superior a 0,78 .

" 1,18 , para $\eta: 1,0 ; \varepsilon:-0,3$.

${ }^{15}$ Pérez Moreda (1982); Nicolau (1989). 
0,46 , según que para la elasticidad-precio de la demanda de productos agrarios $(\varepsilon)$ se acepten valores entre $-0,3$ ó $-0,5^{16}$.

La comparación de los resultados estimados para $\varphi$ con los que requeriría la reducción de la proporción de mano de obra empleada en la agricultura, sugiere que la dotación de capital y tierra por trabajador, así como la eficiencia de la agricultura en el largo siglo xix español (es decir, lo que subyace detrás de $\varphi$ ) no lograron traspasar el umbral que hubiese facilitado la liberación de mano de obra por parte de la agricultura, pero la ocasión se encontraba próxima como nos muestra la experiencia histórica entre 1910 y 1930.

En efecto, el mismo ejercicio efectuado para el período 1910-1930, revela que mientras el valor de $\varphi$ requerido para liberar mano de obra debería superar 0,98 , el valor real de $\varphi$ alcanzó 1,36 , lo que permitió superar también el valor exigido $(1,32)$ para que se produjese un declive absoluto de la mano de obra empleada en la agricultura ${ }^{17}$.

Más aventurado es intentar averiguar si el aumento de (que equivale a una elevación del producto por trabajador) en el período 1800-1910 se debió a una mayor dotación de tierra y de capital por activo empleado en la agricultura, o, si, por el contrario, se debió a una utilización más eficiente de la mano de obra. No disponemos de información sobre el aumento de la superficie agraria entre 1800 y 1910, a excepción de las conjeturas realizadas por Garrabou y Sanz acerca del crecimiento de la superficie cultivada ${ }^{18}$. Esta lo hizo más rápidamente que aquélla, porque, en parte, aumentó mediante la roturación de tierras de pastos. Así, pues, si se aceptase el crecimiento de la tierra cultivada como un indicador de la expansión de la superficie agraria se sesgaría ésta al alza. Tampoco disponemos de datos acerca de la evolución del capital en la agricultura.

Podrían realizarse distintas suposiciones. Por ejemplo, que la dotación de capital por trabajador resultó ser estable a lo largo del siglo XIx, con lo que la tasa de crecimiento del capital equivaldría a la de la mano de obra. Alternativamente, podría suponerse que la razón capital/producto no varió a largo plazo en la agricultura, y, por tanto, el crecimiento del stock de capital sería igual al del producto agrario. En el segundo caso, el crecimiento del capital seria mayor, pues mis resultados sugieren un aumento del producto por trabajador en la agricultura. He preferido utilizar el límite superior. La razón de sesgar al alza tanto el crecimiento del factor tierra como del

${ }_{16}^{16} 0,73$, para $\eta: 1,0 ; \varepsilon:-0,3$.

${ }_{17}$ Estos cálculos los he realizado tomando $\beta: 0,5 ; \eta: 0,7 ; \varepsilon:-0,5$. Los precios relativos agrarios $(-0,45$ por 100$)$ se derivan de París Eguilaz $(1943) ; \pi L(0,69$ por 100) y $\pi L_{a}(-1,01$ por 100), de Nicolau (1989); $\pi N$ (0,84 por 100), de Pérez Moreda (1985); mientras $\pi Q_{a}(0,85$ por 100$)$ e $y(1,54$ por 100$)$, de Prados (1988), y $\pi D_{a}(1,20$ por 100$)$, de Prados (1988) y Tena (1989).

${ }^{18}$ Garrabou y Sanz (1985). 
factor capital obedecen a mi propósito de establecer hipótesis que favorezcan un crecimiento extensivo de la agricultura y reduzcan al mínimo posible el aumento de la eficiencia en el uso de los recursos. Queda por resolver el problema de las proporciones del capital y la renta de la tierra en el valor añadido de la agricultura. Puesto que he aceptado un valor de 0,6 para los salarios, habrán de repartirse el 0,4 restante. Una manera adicional de sesgar al alza la aportación de los factores al crecimiento del producto agrario sería asignar un peso mayor al que crezca más deprisa. En este caso, sería el capital. Así, he supuesto que la ponderación del capital sería de 0,3 y la de la tierra de 0,1 , distribución a todas luces exageradamente favorable al capital. La tasa de crecimiento imputable al factor tierra sería de 0,47 por 100 , mientras la del capital, de 0,98 ó 0,91 (según $\varepsilon:-0,3$ ó $-0,5$ ). Así, de acuerdo con la expresión [7], $r=-\left(\varphi \cdot \pi K_{a}+\tau \cdot \pi T\right)$. De donde $r$ sería igual a 0,189 ó 0,14 (según $\varepsilon:-0,3$ ó $-0,5$ ), esto es, la productividad global habría contribuido entre un 15 y un 19 por $100\left(100 \cdot r / \pi Q_{a}\right)$ al crecimiento del producto agrario entre 1800 y 1910 , en tanto la mayor dotación de capital habría significado de un 28 a un 30 por 100 del incremento.

$\mathrm{Si}$, alternativamente, se prefiere explicar el aumento del producto por trabajador en términos de un mayor volumen de capital y tierra por activo, de una parte, y de la productividad global, de otra, los resultados de este ejercicio conjetural apuntan a que la elevación moderada del producto por trabajador masculino en la agricultura española a lo largo del siglo $(0,23-0,16$ por 100 , según adoptemos $\varepsilon$ : $-0,3$ ó 0,5$)$ se debió en un $30-36$ por 100 a la mayor productividad de los factores de producción, y un $64-70$ por 100 a una dotación superior de tierra y de capital (éste sólo 55-59 por 100) por activo empleado.

\section{BIBLIOGRAFIA}

Anuario Estadístico de España (1923), año X, 1923-24, Madrid, 1925.

Carreras, A. (1984): "La producción industrial española, 1842-1981», Revista de Histo. RIA ECONÓMICA, vol. II, núm. 1, pp. 127-157.

- (ed.) (1989): Estadísticas Históricas de España. Siglos XIX y XX, Fundación Banco Exterior, Madrid.

Chenery, H., y Syrquin, B. (1980): «A Comparative Analysis of Industrial Growth», en R. C. O. Matthews (ed.), Economic Grouth and Resources, Macmillan, Londres, pp. 223.257.

CRAFTs, N. F. R. (1980): «Income Elasticities of Demand and the Release of Labour by Agriculture during the British Industrial Revolution», Journal of European Economic History, vol. IX, núm. 1, pp. 159-168.

- (1987): «Cliometrics, 1971-1986: A Survey», Journal of Applied Econometrics, vol. 2, pp. 171-192. 
Fralle, P. (1985): “Crecimiento económico y demanda de acero: España, 1900-1950», en Martín Aceña y Prados de la Escosura (eds.), pp. 71-100.

Grupo de Estudios de Historia RuRAl (GEHR) (1987): «Un índice de la producción agraria española, 1891-1935», Hacienda Pública Española, núms. 108-109, pp. 411-422.

Lluch, C.; Powell, A. A., y Williams, R. A. (1977): Patterns in Housebold Demand and Saving, Oxford University Press, Oxford.

Martín Aceña, P., y Prados de la Escosura, L. (eds.) (1985): La Nueva Historia Económica en España, Tecnos, Madrid.

NADAL, J. (1987): "La industria fabril española en 1900. Una aproximación», en J. Nadal, A. Carreras y C. Sudrià (eds.), La economía española en el siglo XX. Una perspectiva bistórica, Ariel, Barcelona, pp. 23-61.

Nicolau, R. (1989): «La población», en Carreras (ed.), pp. 49-90.

París Eguilaz, H. (1943): El movimiento de precios en España, Consejo Superior de Investigaciones Científicas, Madrid.

PÉrez Moreda, V. (1982): «La población activa española y su distribución sectorial de fines del siglo xvin a $1877 »$, Memoria del Banco de España, Madrid.

- (1985): «La modernización demográfica, 1800-1930. Sus limitaciones y cronología», en N. Sánchez-Albornoz (ed.), La modernización económica de España, Alianza, Madrid, pp. 25-62.

Prados de la Escosura, L. (1982): Comercio exterior y crecimiento económico en España, 1826-1913: tendencias a largo plazo, Banco de España, Madrid.

- (1985): «Las relaciones reales de intercambio entre España y Gran Bretaña durante los siglos xviII y xIX», en Martín Aceña y Prados de la Escosura (eds.), pp. 119-165.

- (1988): De Imperio a Nación. Crecimiento y atraso económico en España (1780-1930), Alianza, Madrid.

TenA, A. (1989): "Comercio exterior», en Carreras (ed.), pp. 327-362.

Vandellós, J. A. (1925): «La richesse et le revenu de la Péninsule Ibérique», Metron, vol. V, núm. 4, pp. 151-186. Versión castellana en P. Schwartz (ed.) (1977), El produc. to nacional de España en el siglo XX. Instituto de Estudios Fiscales, Madrid, pp. 94-138. 